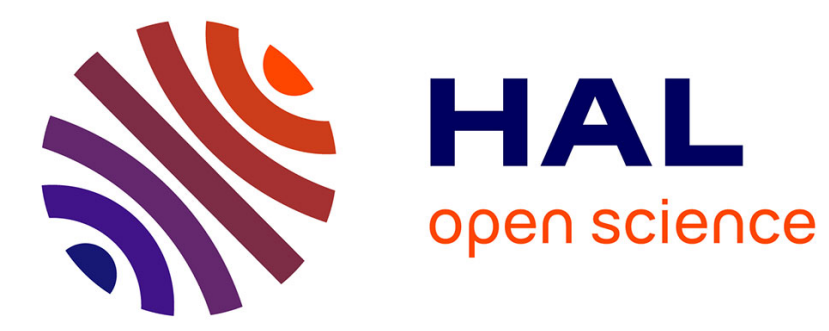

\title{
La céramique incisée à méandres symétriques du Pont du Diable (Aniane, Hérault)
}

Jules Boudou, Jean Arnal, André Soutou

\section{To cite this version:}

Jules Boudou, Jean Arnal, André Soutou. La céramique incisée à méandres symétriques du Pont du Diable (Aniane, Hérault). Gallia - Fouilles et monuments archéologiques en France métropolitaine, 1961, 19 (1), pp.201-218. 10.3406/galia.1961.2321 . hal-01926178

\section{HAL Id: hal-01926178 \\ https://hal.science/hal-01926178}

Submitted on 25 Feb 2020

HAL is a multi-disciplinary open access archive for the deposit and dissemination of scientific research documents, whether they are published or not. The documents may come from teaching and research institutions in France or abroad, or from public or private research centers.
L'archive ouverte pluridisciplinaire HAL, est destinée au dépôt et à la diffusion de documents scientifiques de niveau recherche, publiés ou non, émanant des établissements d'enseignement et de recherche français ou étrangers, des laboratoires publics ou privés.

\section{(1) (1) $\$$}

Distributed under a Creative Commons Attribution - NonCommercial - NoDerivatives 44.0 


\section{NOTES}

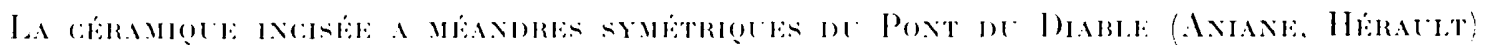

Le petif village de sainl-Jean-de-Fos (Herault est bien commu pour ses ateliers de potiers qui. a la période hisloriefue. du xace

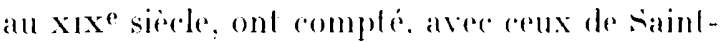

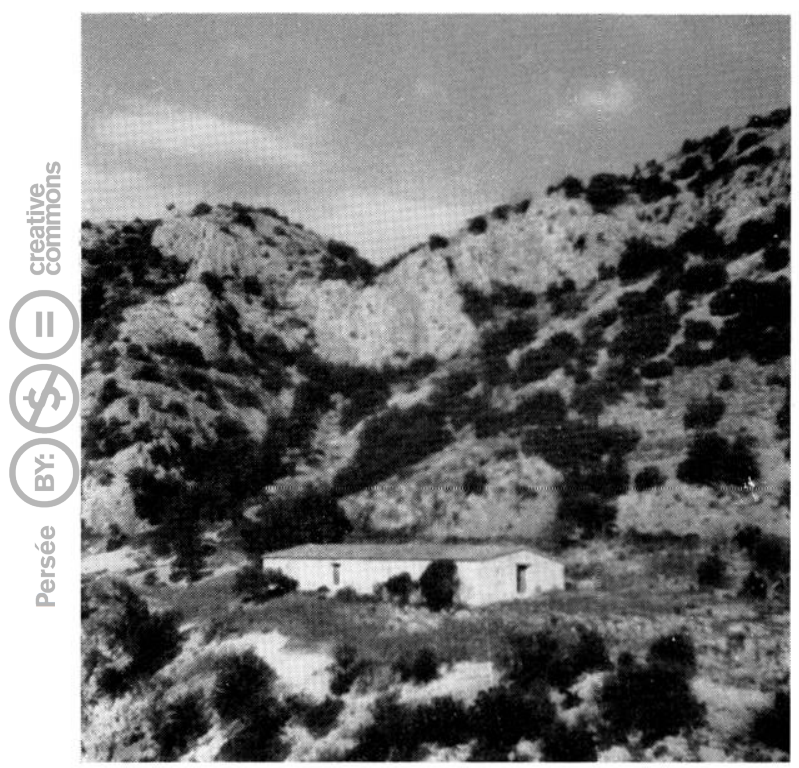

lïg. 1. Vue du grisement à partir du Pont du Idable. I.a station se silue all-rlessus de langle watuche du toil de la berrerere.

Papoul (Aude) et Saint-()uentin-la-loterie (Gard): parmi les plus anciens et les plus actifs du Lanquedor méditerranéen ${ }^{1}$. II semble. loulefois. a en juger par les vestiges archeologiques que nous arons décourerts dans les environs immedials de celle localite. que les

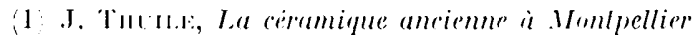

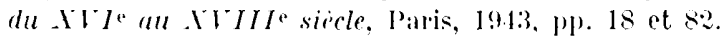
du xie siecle. St-Jean-he-los comptail fo fours a poterie. orjolierse accilans de saint-Jean-de-lios n'ont été que des lointains descendants de potiers protohistoriques installés sur les memes lieux ou presque, au débul du dernicr millénaire avant .J.-C.

Ia station est siluée a l'endroit on l'lléraull. apres avoir creuse des gorges profondes dans les garrigues de la bordure sud du Massif rentral, débouche brusquement sur la plaine languedocienne, précisément a la sortie mème du défile rorheux, sur la rive gauche du fleuve collier. Pour s'y rendre, on peut partir soil de sianl-Jean-de-Fos, soil d'Aniane. A 300 metres de la premiere commune et a 3 kilomedres de la seconde, on arrive sur le Pont du I liable d'ou a été prise la photographie (tig. 1) 1 200 mitres au Nord-Est du pont se Irouve une bergerie, all bas d'une penle d'óboulis que dominent des falaises hautes de bo melres. Le gisement s'alage sur loute la hauleur des éboulis ef est coupe par un thalwegr récent dont les eaux sauvages entrainent des débris de poterie jusque dans la riviere. On trouve aussi des vestiges d'habilat sur le plateau au bord mème de la falaise.

(2) Terme languedocien employé dans les textes du xin et du xvme siecles icf. Thinl, op. cil, passim! pour designer les poliers. L'orjolier est le fabricant d'mrols, c'est-i-dire de cruchons. I.e mot vient du latin urceolus (cf. Wr. von Wartburg, Flill, s. v. urceolusKirugi.

(3) Le Ponl du Diable, nom desigrnant le pont sur llérault qui fut bati dans la premiere moitic du $\mathrm{XI}^{\circ}$ siecle, pour faciliter aux pèlerins lacces de SaintGuihem-le-Desert (ef. J. Vandeny-Ranot, Le Pont du Thiable, dans Congres Archeologique de France, 1951l. 1. 181. Carte d'Etat major, I odive feulle XXVI-43 all 150.000 . Communt d'Aniane. al 3 millimetres an nord du pont. 
Celte posilion accrochée sur une penle raide n'était pas favorable a l'elablissement d'un village. mais sans doule les habilants protohistoriques onl elé allires par les couranls ascendanls de la falaise. properes a actirer le lirage des fours de poliers.

Cous arons pralique quelques sondages. mais la plus grande quantile des lessons recueillis l'ont ate en surface. En oulre. (i. B3. Arnal a trouve une fibute dans le thalwer (lig. 2). el a eu l'amabilite de nous la remellre. Ie sombage principal nous a dommé la slal igraphie suivante:

30 rentimelres d'éboulis de pierraille mélangée de lerre.

5) centimidres de foyers noirs, cendres mélangées aux lessons de polerie. Lin haut se I rouvail le vase entier (tig. ¿), 1) partiellement dégagé par l'érosion. I mi-hauleur. grisaient des lessons annelés ou godrommís frilled ware de Nancy K. Sandars). I la base. se Irouvaient des lessons orne's de larges cannelures of d'impressions cupulaires aree le lesson orne de méandres symetriques (lig. 11, no 3 ! Il y avail aussi des fusäoles el des houdins d'argile.

le sol repose sur des pierrailles of do sable apporte. sur lequel des vesliges de leote sonl malerialisés par des trainées hlanches. Apres avoir avancé a l'horizonlale sur $1 \mathrm{ml}$. 5), nous nous sommes heurlés at un mur de pierres irrégulieres soudés par le feu, durei au point qu'il est difficile de l'entamer au pic. On a l'impression d'alre sur les restes d'un four de potier, bien qu'il manque la "grille" de lerre refractaire que l'on est habilue a trowrer dans de lelles constructions.

less sondages ol le rambssiger an sturlace nous onl permis de recueillir un assez important malériel archeologique, qui comprend:

1) De nombreux lessons de céramique que nous analyserons par la suile. parmi lesquels il comvient de relever quelques pieces manqueres aux parois lordues el boursoullées qui sont visiblement des rebuls de fabricalion (liw. 7,2 .

2) Des boudins anmulaires d'argile cuite

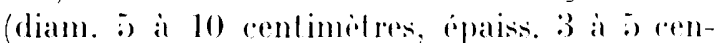
limelress.
3 Jes calels plals el lisses qui semblent. avoir servi de lissoir pour poleries.

1) Des os d'animand provenant de debris de. aluisine.

5) Des fusäoles an nomber de six. dont

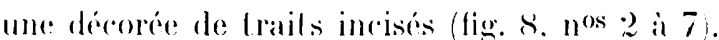

(i) line dibule de bronge a ressort en arbalide are arrondi de secelion ovale el pied relevé lermine par un boulon eoniques (lone. ¿) entimbles; fig. :

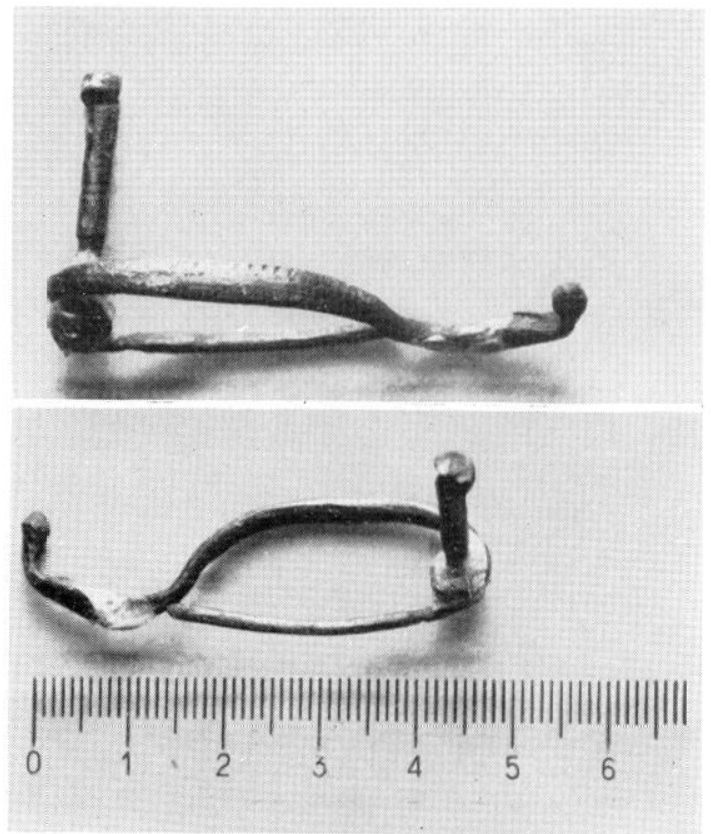

lï. :. Fibule hallstattieme.

$\therefore i$ les dobris de ruisine a les fusatioles indiquent la présence d'un habilal, les boudins d'aregile. les lissoirs et les pièers manquees permetlent de préciser que la fabricalion de la polerie alail une des principales orempalions des habilanls. L'enderoil bait diallemes forl

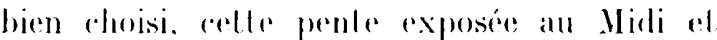
bien abritere des vents du Norrl, alail lris favemable a l'abablissement d'un groupe humain el les fours de poliers, comme nous l'arons déja dit. benéficiaient pour leurs foyers d'un lirage exerptionnel. l'examen de la céramique permel, dans une cerlaine mesure de delerminer la duree de lonerupalion du sile ot la dale approximalive du premier chablisstmenl. 
I. Ouelques tessons. tris peu nombreux. peuvent itre classés a la fin de l'époque romaine ou au haut Ioven Age. Ce sont en particulier :

a) un rebord a bourrelet arrondi rejeté vers l'extérieur. présentant à l'intérieur une légère gorge destinée à soulenir un couvercle. Paite gris-noir. bien épurée bien cuite, douce au loucher (fig. 3, 1) ;
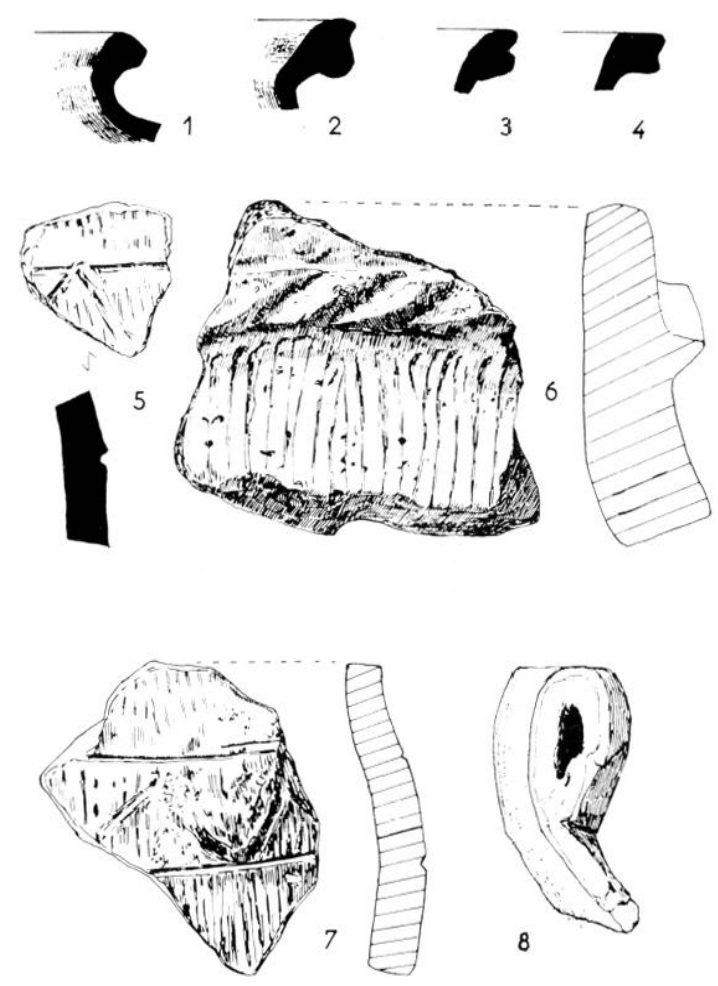

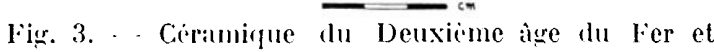
de la fin de liporpue romaine.

b) Trois rebords également éversés mais donl le bourrelel supérieur est articulé en deux parties par un sillon médian horizontal. L'intérieur du rebord présente. comme sur le lragnent précédent. un léger ressaul pour recevoir le couverele. Ces rebords appart iennent a l rois vases différents, a pale grise ou gris-clair. Ils se rallachent i une céramique tress répandue ¿ l'Ouest du Massif Central ${ }^{4}$, qui a duré dans celte région de la période gallo-romaine au Ioven Ige (fig. 3. nos 3 i i l.

II. Alors qu'aucune céramique gallo-romaine de bonne époque (lerre sigillée n'a été observée, par contre certains tessons peurent ètre allribués a l'époque de La Táne:

a) un fragment de polerie assez fine a décor exéculé au peigne fin. Pàte rougeàtre bien épurée. Ce lesson se place probablement i La Tene III ;

b) deux fragments de vases de grandeur moyenne combinant te décor peigné avec lo motif ondulé. Pàte grossierre. mal épurée. l riss éloignée de la céramique phocéenne dont ils peuvent ìtre une dégénérescence. Ces lessons sont aussi très différents de la poterie grise à décor ondé de La Tíne III. Ils représentent sans doule une céramique indigine plus ancienne mais de facies non hallstallien (fig. 3. nos i) et 7);

c) Irois fragments d'une grande jarre a panse enlierement peignée exlérieurement et a l'inlérieur. ornée au sommet de l'épaule d'un fort cordon horizontal is incisions obliques simulant une lorsade (diamilre au niveau du cordon : environ 20 centimètres, épaisseur des parois : 2 cm. 1). Il s'agit d'un récipient de tradition hallstallienne qui a emprunté aux dolia méditerranéens. importés dans le Midi de la France dies le rre siecle ${ }^{5}$, le décor peigné (fig. 3, 6);

(4) Cette forme apparail dans l Averron dis láponue gallo-romaine, par exemple dans le fanum du combalou a Roruefort (collections de la societé d'études Roquefortaises). Le nombre infime de framents de ce type dans le gisement de st-Jean-de-los, oir, par ailleurs, la céramique abonde, semble indiquer que les vases correspondants n'ont pas ete falripues sur place : peut-ìtre proviennent-ils de lat rigrion de Millau, oì un four fabricant ces poteries fonctiomait pris de la ferme du Vaubert fommune de Ia Roque-Ste-Narguerite: fin tout cas les trois framents du pont du Diable sont. jusqu'a present. a notre connaissance. les exemplaires les plus méridionatux de cette ciramique caractiristique.

(5) Ph. Hitixi, Les Origines de Narbinne, Nablome, 1937 , p. 29:7 sfr. Il est interessant de remarifuer a ce propos, que ce decor peigne s'est conservé sur les jarres jusquen pleine epoque romaine, puisquion le retrouve, associc au dicor ondule, sur les urnes qui servaient a la distillation du bois de pin sur les cirands Causses. Ial présence de ce decor, à St-Jean-de-Fos. sur une jarre de tradition hallstattienne, semble contirmer l'hypothese que nous arons formule par ailleurs A. Sol Ton. Latelier de resiniers gallo-romains. de P'uech-Maryue (Cone de La Cresse. Aleyrom), dans l'allas, YIII, 1959, p. 85-99\%, a salvoir que ces momes 
d) fragments damphore a livere arrondie, repliéc sur elle-meme vers lexterieur. Paile rouge asser\% grosiciore (lig. 3). si ;

e) Vase légerement bi-conicfure it carime adourie poneluee diune ligne lorizonlale d'impressions triangulaires. fond plat (lig. i). $1 !$.

III. En dehors des reramiquess énumérés ci-dessus. qui romposent deux groupers hien
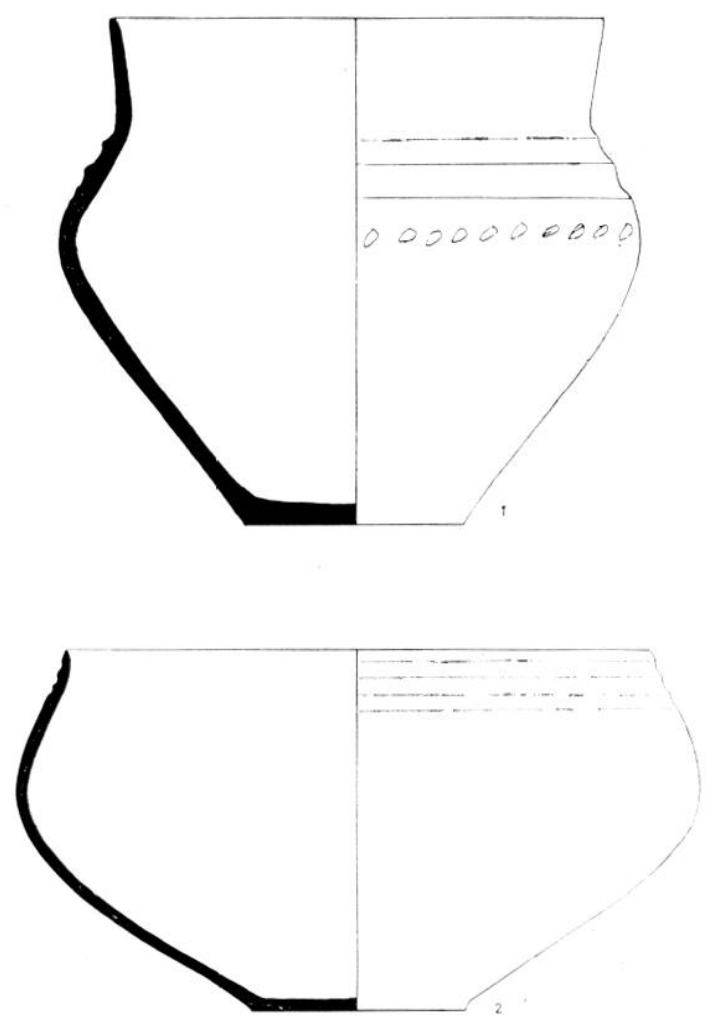

fin. 1. -. Dend vases hallstattiens,

distincts. la grande masse de la poterie du Pont du loiable est constiluere far des lessons le remamigue hallstalliemme. parmi lesquels on peul distinguer les formes al les déeors suivanls:

1. Formes:

a) lime biconique it carene arrondie el rol lewerement incline, orne sur lépaule.

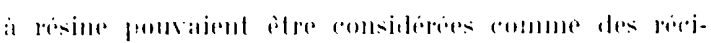

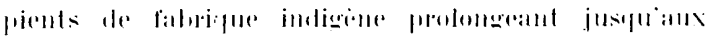

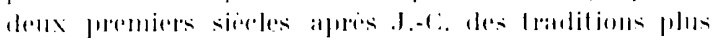
ancirtmins. en haul. de deux larges anmelures horizonlales, en bas. d'une ligne d'impressions ovalaires; font plat (tig. 1, I et fig. 5. 2). Ce récipuent est brun aver des roups de leu noirs; sal pale est lustren mais asser encosiciere.

b) bols arrondis ou largemenl ourerls. de loules lailles, ornés sur leur parlie superieure de cannelures horizontales ; londs plals (fig. li. 1. el fig. 10. 3):

c) assietles plates a bords legerement rentrants (fic. o. . 3 el fiw. 6. 1 :

d) assietles-eouvereles a exlerieur ruguedux el inlérieur lissé. ornées de annelures eoneenlriquess. Les bords el les fonds manquent; aucun exemplaire n'a pu itre reconslitué (fic. (i) nos 2 el 3 );

e) urne a col rylindrique el bord dégagé ornée de cannelures concentrigues sur l'épaule (fig. 7. 1 ! ;

f) urne a bord rylindrique el bord ciase orné sur sa parl ie supérieure de deux rammelures romerentriques. Certains onl des profils anguleux liw. 7.2 a 6 or 8 : :

(1) urnes biconiques ornés sur lépaule de triangles imprimes on de rannelures (lig. 8. $1:$;

h) bols biconiques a carine on arrondis. ornés sur le eol ou sur la rarene de lrails incises avanl cuisson (fig. 6) 6-7 al lig. 10. 1 (1) 2 .

B. Hecors:

a) a impressions rondes. ovales ou lriangulaires. disposées sur l’épaule des vases el sur les cordons silués a la base des cols (fig. 1. 1 (a) fig. S- 8-11);

b) a cammelures forles placéses horizonlalement sur l'épaule des vases (lig. s. s) :

c) i camnelures legeres. (ie diecor mol

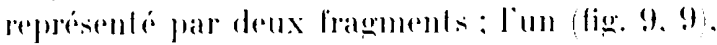
a rammelures asser larees el longues. oblicgues placees sous la rarene au-dessous de rammelures

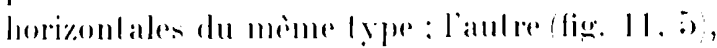

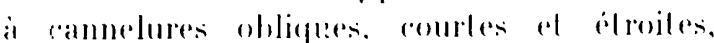
disposies stur une rariene arromde all-dessolls d'une ligne de points en creax al d'un déore incise :

d) incise: les molits. toujours enemeitriques. sont fails soil de lignes horizonlales paralleles.

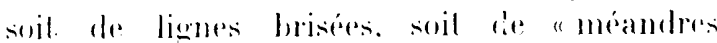
symelriquess cnous reviendrons un peu phus 


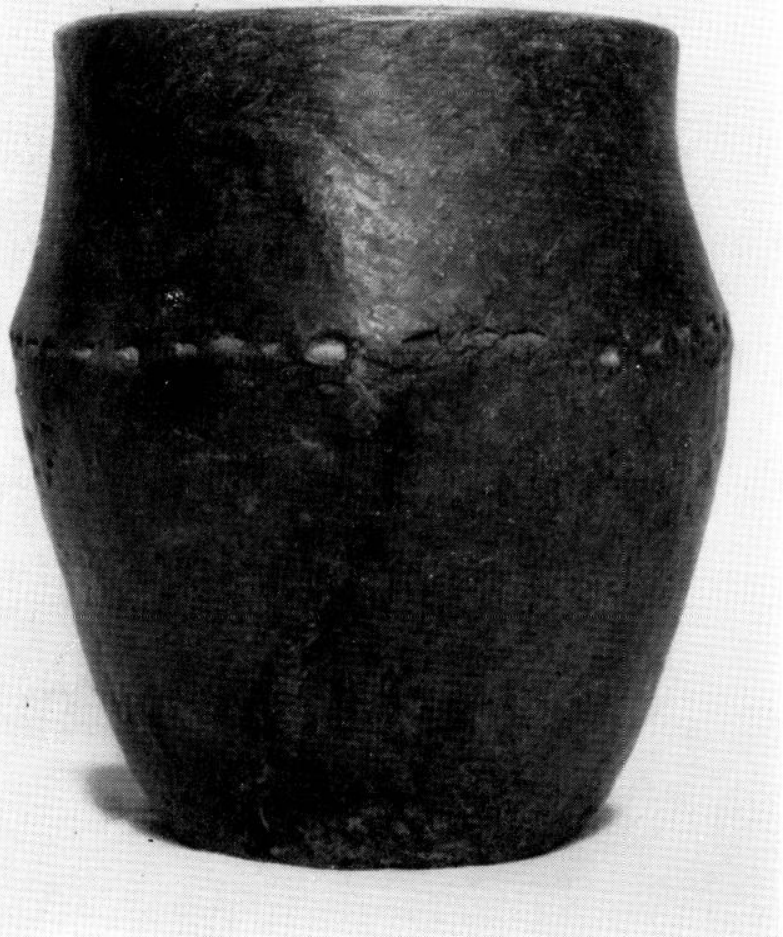

1

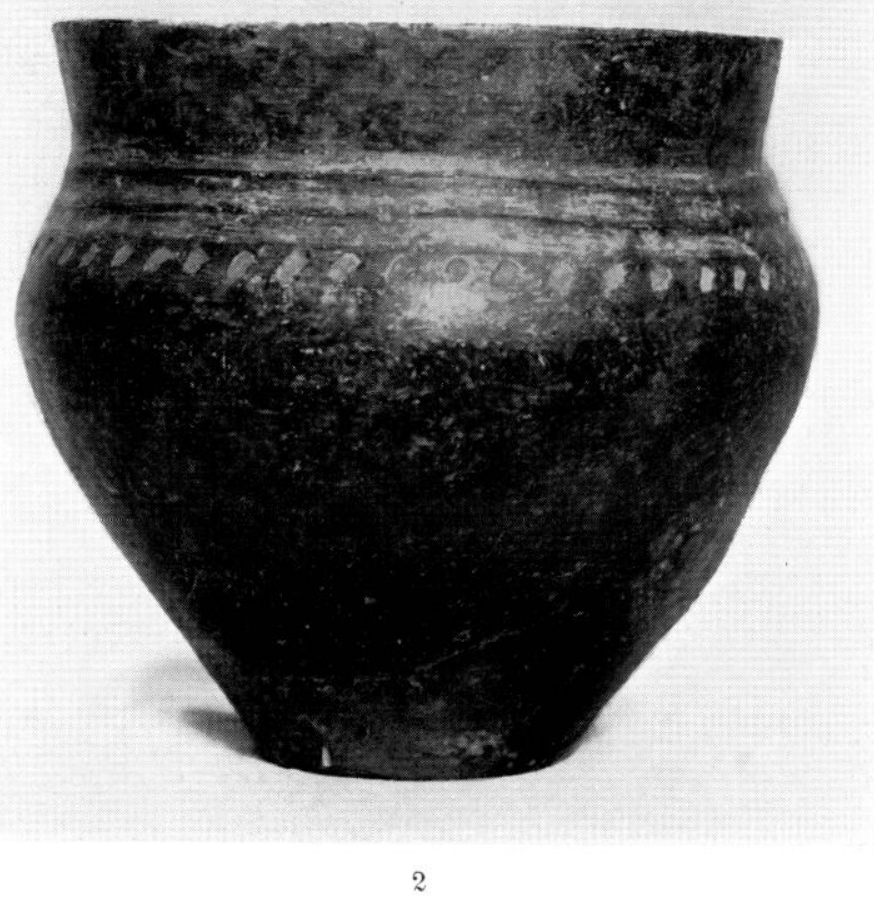

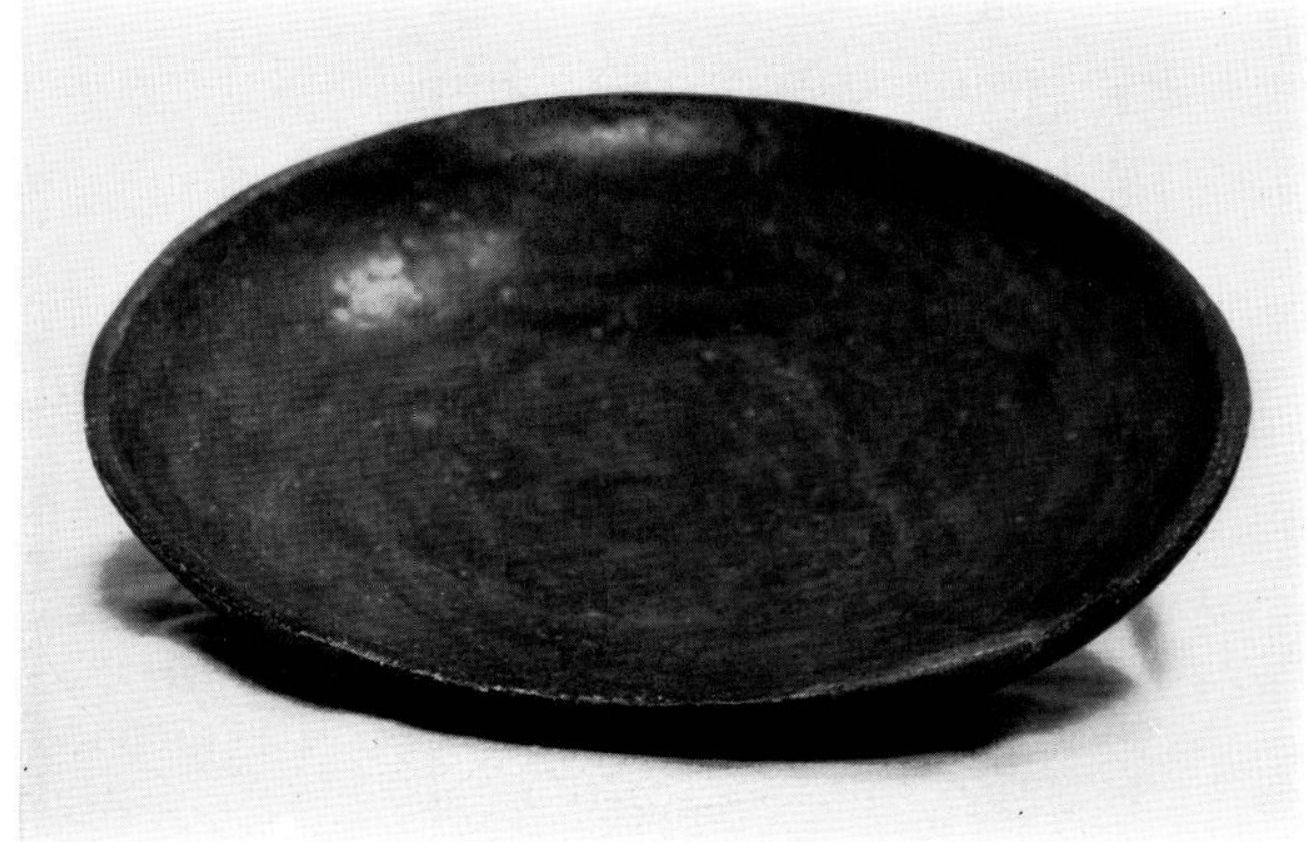

3

lize. 5. diramique hallstattiemme el post-hallstatlieme. 

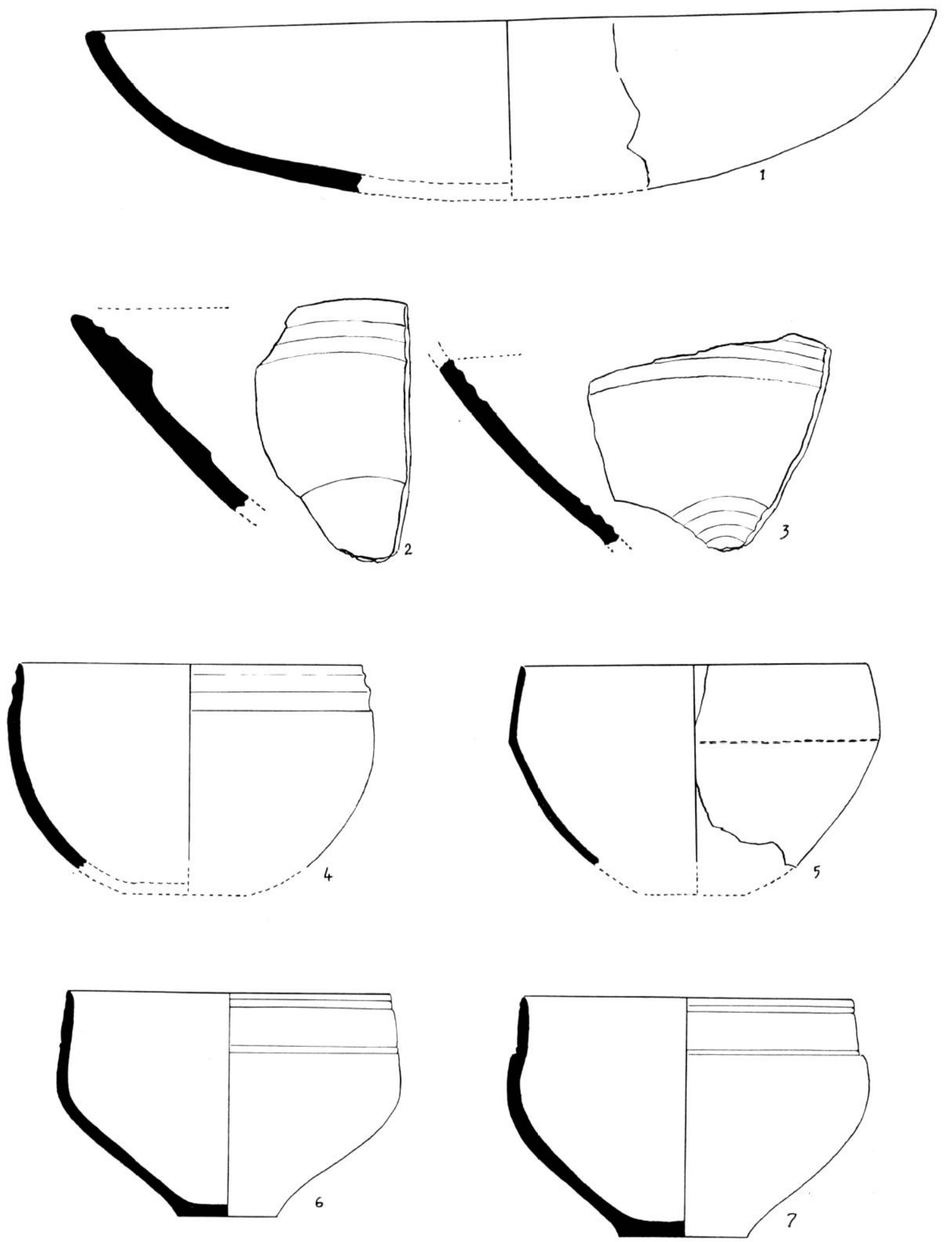

Fig. 6. - Plat, assiettes-convercles et hols. 
loin sur ce decor particulier. Ces motifs sont tracés au double el au triple trait. I'incision. loujours praliquée avanl cuisson. est plus ou moins profonde; parfois. nolamment sur les céramiques a pàte brun clair, elles apparailront en noir sur la surface mème de la paroi : dans ce cas elles ont été lracées arec une pointe mousse agissant comme un brunissoir.

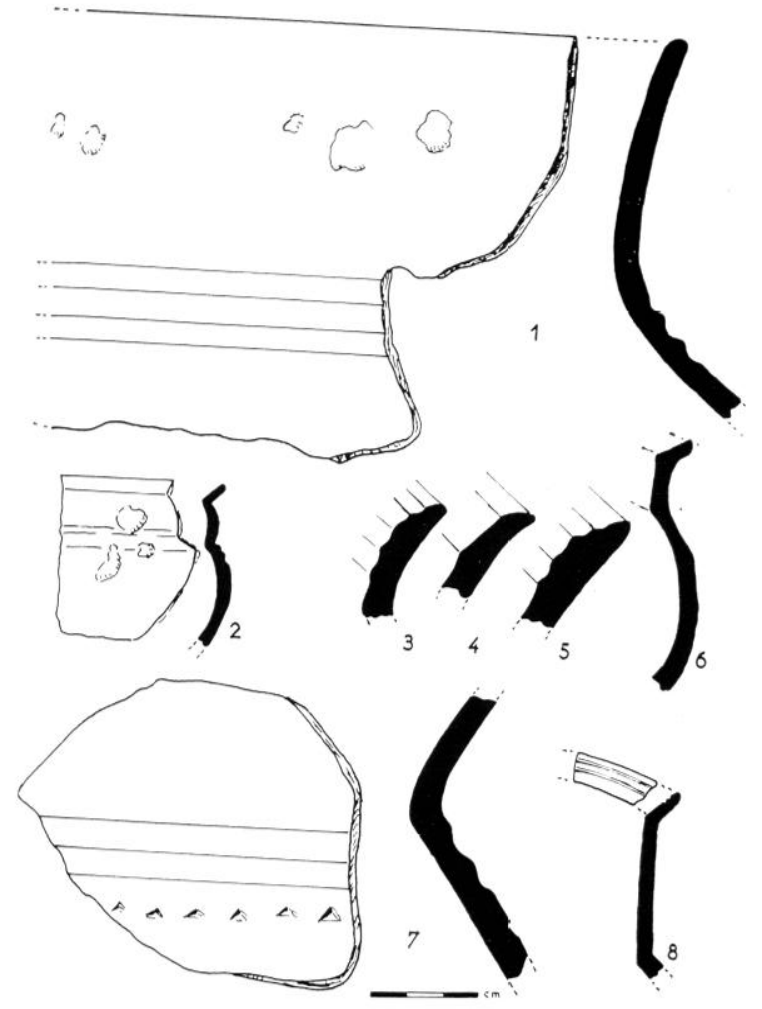

fin. 7. Ditails de quelques hords ou cols.

Pour aulant qu'il est possible d'en juger par l'analyse des formes, la plus grande partie de celle céramique semble appartenir au débul du Premier Ige du Fer (Hallstalt I

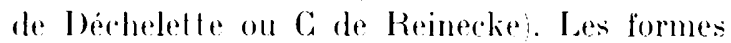
n-e présenlent les profils nels. les bords degages. les carines asse\% bien marquées les facelles el les cannelures qui rappellent encore les I radilions des Champs d' ' rnes III, mais qui doivent se placer à une époque plus tardire, qui se prolonge sans doute jusqu'a la fin de la période hallstallienne fibule à ressort en arbaliete el fonds annulaires). Toutefois. il est certaines formes $\mathrm{f}-\mathrm{i}$. en particulier l'urne a col relindrique. leurne biconique a col a entonnoir. le bol biconicue, el surtoul certains décors "méandres sprmélriques" et rannelures légeres disposés obliquement qui permetlent de rattacher indubilablement une fraction importante de celle céramique a la période précédente (Bronze Final III ${ }^{6}$. L.e méandre symétrique (pour employer l'expression de V. Gessnerg est une figure

(6) Nous adoptons le systime chronologrique utilise par X. K. Samdars dans son live sur les Cibilisalions de l'Age du Bronze en France (Bronze Age Cullures in France, Cambridere, 1917 . qui traite principalement des dernieres phases de celle periode, du xm" au

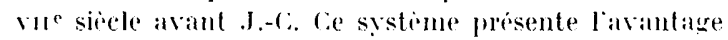
d'englober dans le Bromze limal les principales itapes de la civilisation des Champs d'l prnes. telles quelles ont ete distingues par 11. Kimmigr. Voici le tahlean de correspondance des systimes chronologingues les plus collums :

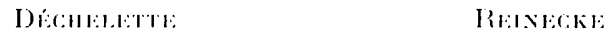

Bromze III Bronze D)

Bronze $\mathrm{N}$ Mallstalt I

Jallstatt I3

Hallstatt It Hallstatt $\mathrm{l}$.

KIsML: SANUAR

C:I I Bromze linial I

r.I II Bronze linal II

C.I III Bromze lFinal III

Ane du fer.

(7) Verena (issixl:k, Die geomelrische ornamentik des spälbrunzezeillichen l'fahlbankreises der sihureiz. larese, 19.16. I.e meandre symetrirue est ainsi caractérisé par lauteur p. 6.3, s. 5: : "Sur un grand nombre de vases provenant de palatittes, d'habitats et re sepultures nous renconlrons un notif incise, constitue par des lignes parallies que nous nommons méandre... I.e miandre a toujours la mime apparence, qu'il soit vu d'en haut ou d'en bas et il n'est pas possible de lui assigner une direction car il n'est dirige ni vers lat droite ni vers la ganche. cette varicti de miamlre est typipue des palafittes suisses de la lin de l'ilge da Bronze. Sa caracteristique principale réside dans sal structure symetrique, qui est essentiellement diffirente de celle du miandre grec et italipue. Ce dernier meandre, qui est asymetrique. puisquil est dirige vers la droite ou vers la gauche, est incomnu en Suisse a la fin de lAge du Bronze. I la famille du miandre a angles droits s 5: appartiennent le meandre a aneles arrondis 553 . le " miandroüle " que uous appellepons plus loin "miandre en II couche "s 51 le miandre

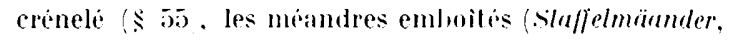
$\$ 56$ et les moandres rectamunaires $\$ 57$. 1,e minulre symetrique apparait en suisse a la fin de la periode de

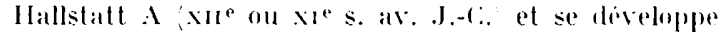
surtout an cours de la prepiode suivante. Il nest mulle- 

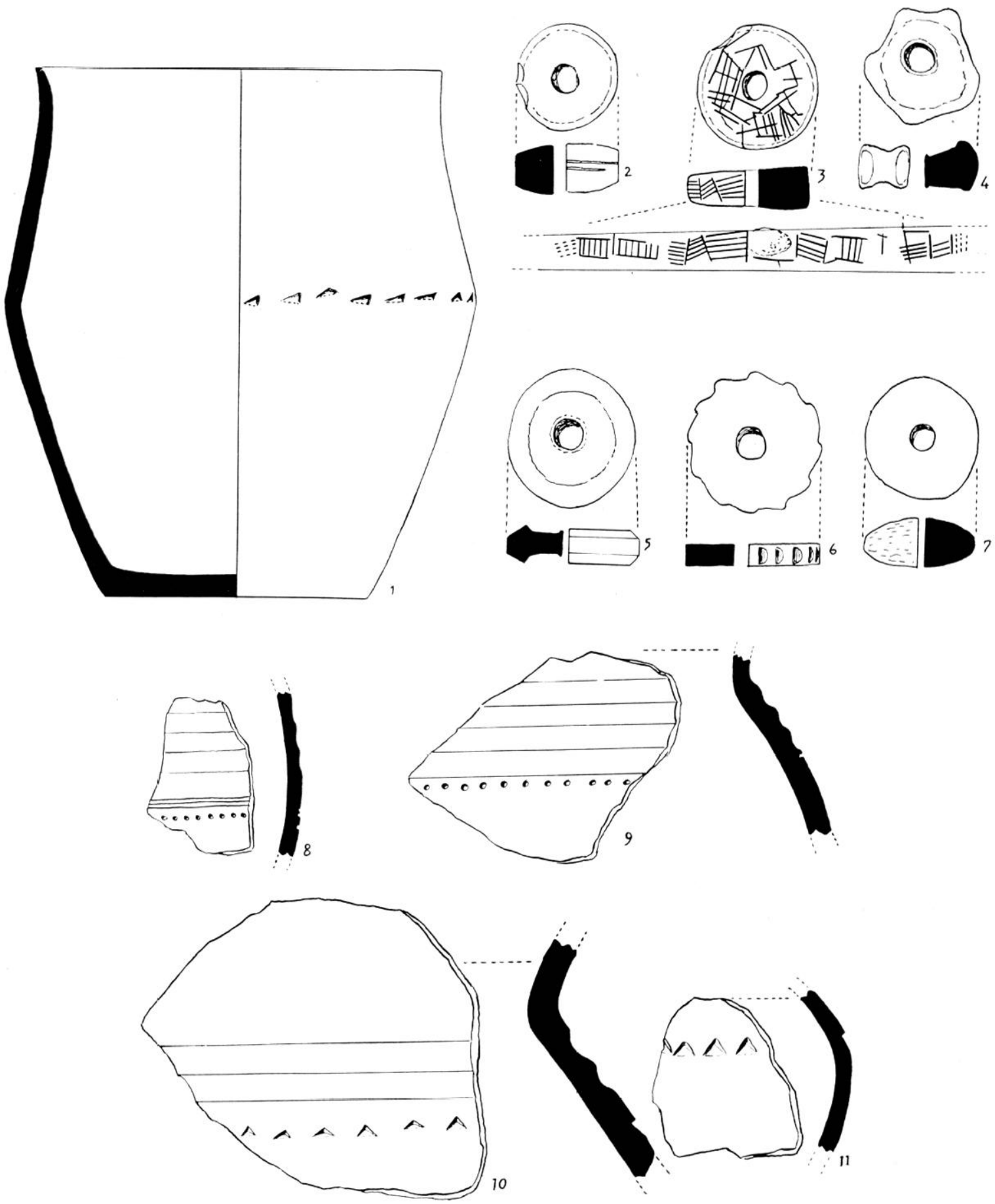

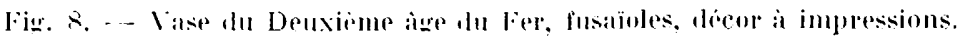


geometrique rlont loutes les parties sont absolument srmétriques par rappert i un axe médian : il est essentiellement different de la grecque ou méndre asymétrique. qui est loujours orientée soil vers la droite. soit vers la gauche. C'est le motif dominant de la céramique incisée du Pont du Iiable, où il apparaît sous forme de méandre à angles droits. simple ou double (fig. 11. nos 1 a 3 ). I es simples motifs gémétriques (xigzags el lignes parallibes) de deux aulres lessons (fig. 9. nos 1 is se classent dans la mème famille décoratives. Or. le méandre symétrique. méandre immobile el equilibre. de struclure slatique. est caracteristieque de la derniere phase des palatilles sulsises el peul ilre consideré comme une verilable forme directrice de lextreme fin de l'Age du Bronzes. Ce molif ne doil alpe ronfondu ni avec les figures asymétriques ni aver les figurations slylisées de personnages ou d'animaux. que l'on renontre sur les reramiques plus récentes el dont les origines. alusi bien que la datation, ont été précisées par X. K. Sandars ${ }^{10}$.

In deuxieme élément décoralif altribuable a celte meme periode du Bronze Final III est constitué par les comnelures légères. qui sont equalement anterieures at la premiere phase de Mailhac"n. Ces rannolures lómires (rilled ware).

ment derive de la grecque imiandre asymetrique: : bien an contraire il est antérieur a elle. la grecpue, en ellet, est incommue a Athenes avant le milieu du xe siecte style grometrique ancien: on he la rencontre ni a lepocpue protogeometripue ni a leporpue submecenienne (ibidem. p. 67 el tix'.

$\alpha$ tommo on pent lo constater sur un fragment de Gresine (figr. 1:. 7 , que nous menlionnerons plus loin, le zigran et les tmils paralleles incises an double on triple trail sont associes sur le mime vase a des motifs derives du meandere symetrigue A. Prons, Flude prehistorique sur lat itawie. specialemenl it lepoque lacustre, chambery, lsio, pl. XX. 1\%.

(9) Die Bromzezeil der trhmerz, cahier idite par li societi suisse de Prohistoire. \%urich 1!56. p. ?0.

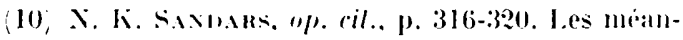
dres asymetrifues et les figurations stylisies sont arisinaires de la Méditeranée orientale et sont parvenus en Ianguedoc par lintermidiare de l'Jalie du simel cometiere prihellénique de tiumes, a partir de la tin dil ville siocle a1s. I.-

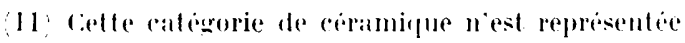

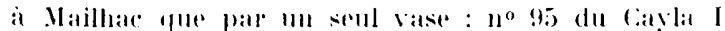

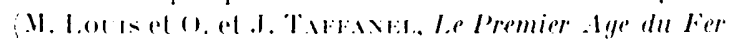
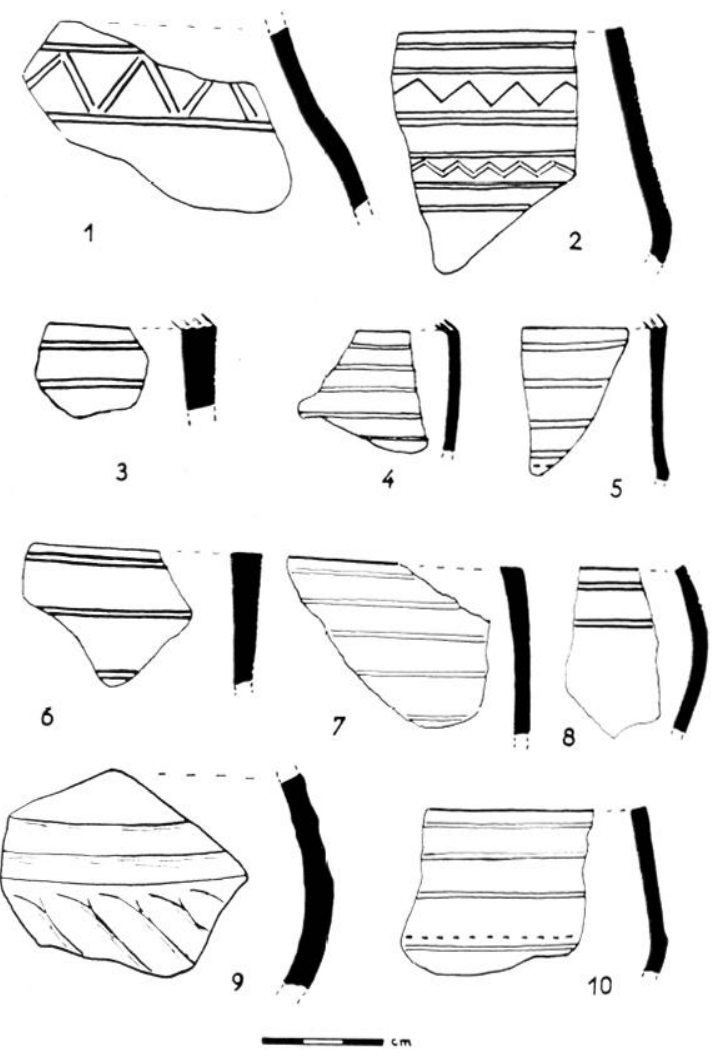

lig. 4. - Decors incisis et cannelures lemines.

que l'on renconlre déja sur les céramiques de l'Europe orientale ef de l'burope centrale antérieures a la civilisation des Champs d'l'rnes, apparaissent dans l'Lst de la France dis le Bronze Final I (C. L. I) et se developpent aux périodes suivantes ${ }^{12}$. Iu Ponl du liable. elles sont datées du Bronze Final III. comme on peut le constaler sur un fragment raracterislique (fig. 8. 5). qui unit. sur un meme base. le méandre symétrique el la cannelure lécrire. Ce tesson. d'importance capilale Irarluil. dans le domaine de la céramique. la fusion qui sest opérée dans le cadre de la civilisation dess Champs d'l'ness entre plusieurs coumants

I.rnguedorien. Bombighera-Montpellier, Is5.). p. s. fix. jx. 1, qui pent ète interprete comme un vestige attarde de la perionle précidente.

l2 X. K. SAxnks, "p. ril., passim. I. auteur itulie la diffusion en france de celte ciramicue caracteristique que nous appelloms i camnelures lighes (leichlgerille Hiare de Holste, rilled ware de X. K. Sintdits. 


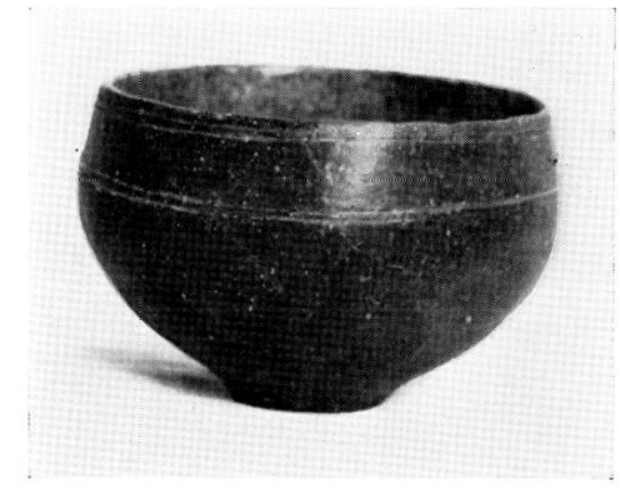

1

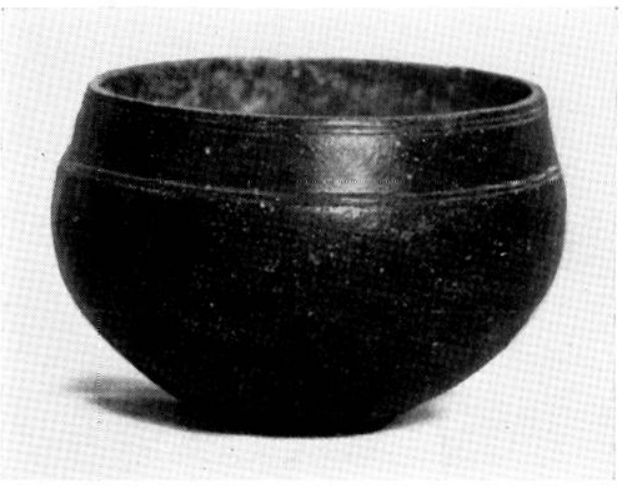

2

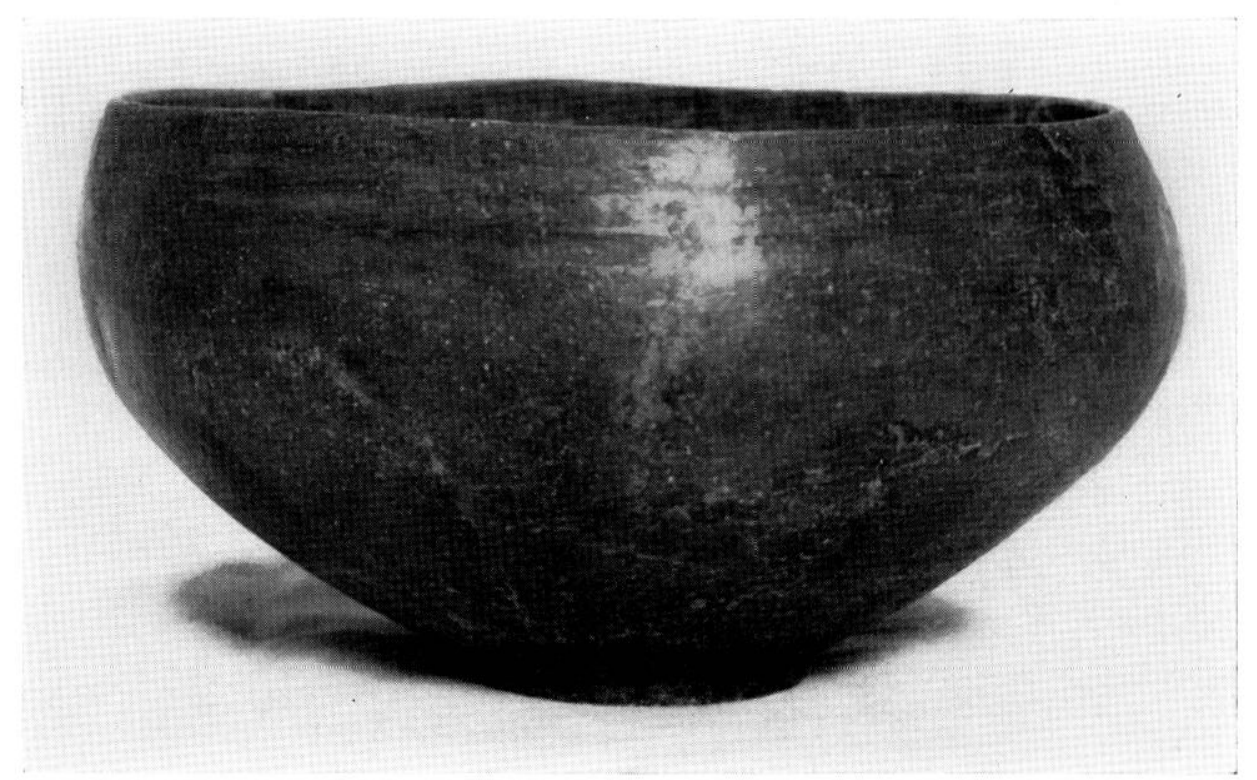

Jig. 10. - Bols ornés de décors incises ou de cannelures.

paralleles d'origines dilférenles. qui converwent et s'amalgament dans le . Midi de la France.

Les minures on cammelures concentriques que l'on relrouve ausis bien sur le bord épuarri des fragments à decor incisé (fig. 11. 2) que sur le rebord du vase a col alindrique (fim. 7,8 ) sont également de la mime epocfue. comme le monl re le fragmenl de la fig. 11. 3. qui présente a la fois ces rainures lacees sur la lranche de son bord rarré el le decor a méandre symélrique. Larticulation savante de la surface supérieure de ces bords a angles vils est en loul point comparable a la multiplication des facelles sur les bords des assielles-courerales (lig. 7,3 a 3 ). Or, on sail que celle prédilection pour les biseaux of les cannelures a l'interieur des rebords est tres caractéristique de celle mème période du Bromze final $11 \mathrm{l}$.

Enlin, un delail particulier s'allache plus spécialement aux framents a decor incise : le bord superieur de prestue lous les vases qui portent re déor est franchement épuarri. Il presente une tranche plate. souvent plus épaisse que la paroi des vases el, dans certains cas. la surface ainsi oblenue est ornée de ces rainures ou rammelures dont nous venons de parler (fig. 11, 3 el 6). Ce bord carré semble eonstiluer un elément lypologique de la cramique du Bronze Final III, puisqu'il est associe, lui aussi. sur un fragment au moins, 
all décor à méandre sprmétrique fie. 11. 3). Ce detail lypique se relrouve non seulement dans d'aulres sites du Midi de la France, par exemple a la grolte de la Chevreris, siluée press de Meyrueis. Lozere fig. 12, 3. mais ausi dans le reste du pays. jusque dans le XortOuest, notamment au Fort-Harrouardit. Il
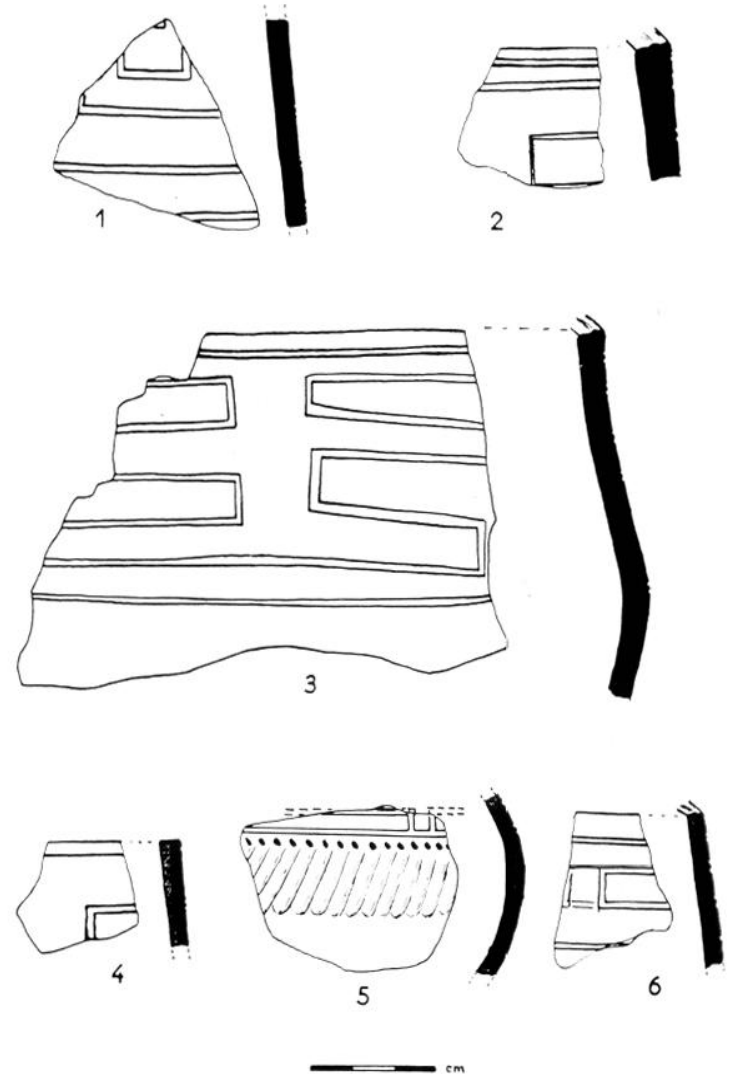

l:igr. 11. -- Miandres șmetriques et comnelures ligeres.

ne s'agil done pas d'une parlicularilé locale a aire de répartition limitée; bien au contraire, le bord plat a angle droil, orné parfois sur sa tranche supérieure d'une ou plusieurs cannelures, peut itre considérć comme un délail caractéristique de la céramique que nous venons d'analyser.

13) Franments deposes au .1 usemu d'histoire naturelle de Toulouse fouilles Trutat:

14. X. K. Savmors, op. cil., p. 273, fị. 77,1 , jet p. 27. tix. $7 x$. 12. Il convient de noter quan fortHarrouard ces hords carres sont associes dans le foyer 43 an dicor a meandre symetrique ibidem, fig. 77,6).
La présence au Pont du Diable, de céramique ornée de méandres symétriques est loin d'itre exceptionnelle, ainsi que l'on peut en juger par la liste des gisements silues au sud dune ligne allant de la kochelle a Genive.

Celte liste se rapporte a une zone comprenant non seulement le Midi de la France mais aussi la Péninsule ibérique, qui, en ce qui
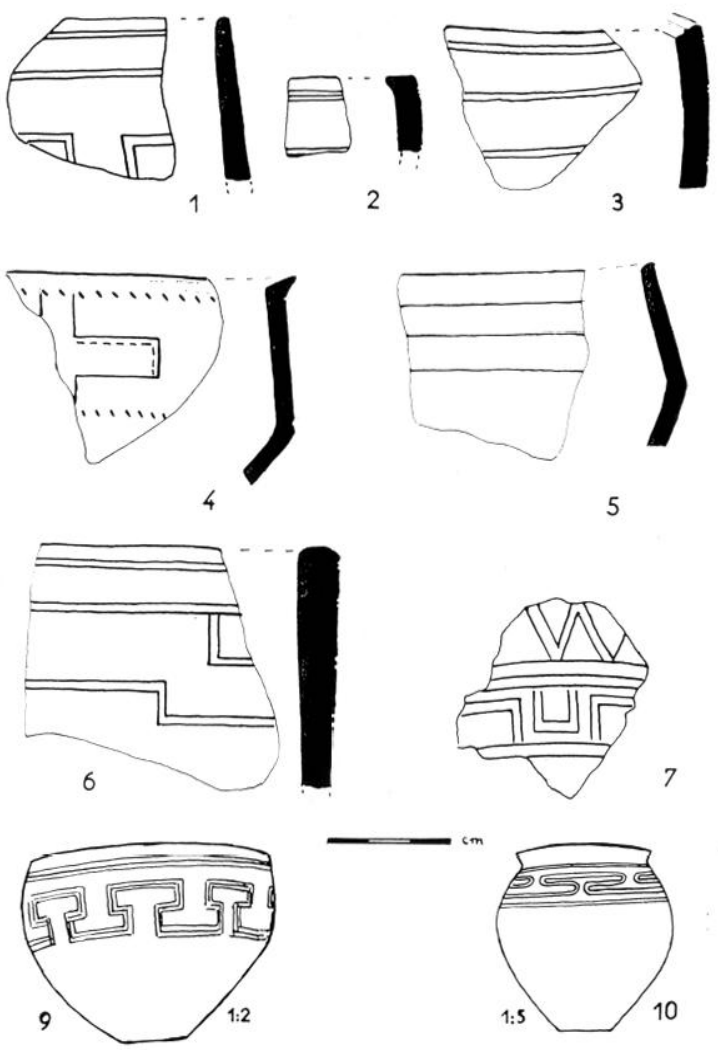

Fig. 12. - Ciramiques de provenances diverses ornces de miandres symitriques.

concerne la répartition de ce molif parliculier, se réduit a la seule Calalogne. Ies dillerentes variélés de méandres symélriques sont figurées en bas de la carte (fig. 13 a-g) el ainsi désignées : méandre angulaire (a), méandre arrondi (b), méandre double (c), méandre crénelé à angles droils ou aigus (d). qui se présente quelquefois en séries emboilées les unes dans les autres (Slaffelmäander de V. Gessner, of. nole 7 ), méandre en "H " couché (e) $)^{15}$,

15. Cost le mandroïle de $\checkmark$. Gessner cef. note 7 qui ne doit pas atre conlomdu arec le motif lu persommage stylise que loon rencontre à I'J Ire du lier, aussi 
méandre rruciforme (j) méandre rechangulaire (a).

Dans l'énuméralion qui suil. les nombres correspondent a des points éeographiques distincls groupes par départements ou provinces (les chilfres de cette liste correspondent a ceux de la fign. 1:3):

1) Sivorks slation du lac du Beurgel. Comme la souligné $X$. K. Sandars. la céramique de res stations. identiques a relle de suisse (palafilles du pays de Bade (Giindlingen) ol de la région du lare de Constance (Singen) ${ }^{17}$ est bien daté de la tin de l'. Ige du Bronze (Mallstall IS de Reineckel. Mentionnons un vase entier de Chatillon orné de méandres arrondis ${ }^{1 s}$ el un fragment de Grésine (fig. 12.7 dont il a éce dejai question a la note 8 .

2) Chandiste lilhomnent: habilal et grolle sépulcrale de Bois du Roc: fragment décoré de méandres a angles droils ${ }^{19}$; contexte imprécis.

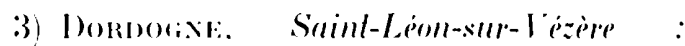
grolle de la Ropue-siainl-Christopher dans le niveau 1). des céramique a méandres a angles droits elo daient melangés a des ceramiques a cannelures lexgeres.

1) Cilloxint. Blasimon: ruisseatu souterain

bien sur la poterie incisie que sur la polerie champlevie à excisions : les baras et les jambes metlement arlicules permettent de distinguer cette schimalisation diun atre humain du mindre en II conchi qui est. selon l'auteur, une simple figure grometrique cl. N. K. SaxDARs, op, cil., p. 319, fig. 95, no 18, 20,21 : ceramique provenant du champ d'urmes d’Agullama, Caladogne. doun tumulus de Cazevieille, llerault ef de la grolle de la lanume-Longue à Lions, Gard .

(It) Bien fue ce motif soit incommu an suisse of que mous mo layous releve que star un seml lessoll no l! de la lister, mous pensons foutefois, justua a nourelordere quil appartient a la famille du meandre simetrifue. Il resulle, semlıle-t-il, de la juslaposilion de denx meandres en 11 couchi qui determinemt, dams lontervalle qui separe deux motifs comtinus, des miandres crueiformes, comme on pent lobserver sur IIn vase de la tombe 17:2 de Millas 0 . 31. fig. 17 de la publication citie plus bas a la mote 39 .

(17) Pelit bol a meandres angulaires fix. 1:. nº du champ d'urnes de Singen 11 . Kinme; et H. Ml:1.,

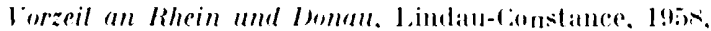
l. (i.3, fị. 71 ;

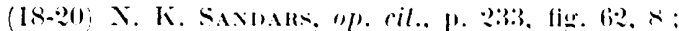
р. 257. lig. 71,1 ; р. 929, lig. $69,7$. des Clusels; un fragmenl de vase biconique orné d'un décor de méandres rectangulaires foollection de M. R. Couste, président de la soc. arch. de Bordeaux).

5. 9) Iriskox,

i) Millau: grotle de Calapade ; un fragment décoré d'un méandre arrondi est exposé all Musée fenaille a Rode\%.

6) Millau: habitat du serre de la ciranede; fragment orné d'un méandre crenele a angles droils ${ }^{21}$ of autres fragments inédils a meandres angulaires.

7) Roqneforl: arrolle des Fées: plusieurs fragments ornés de méandres anqulaires (Musé de Roquefort).

8) Sainl-Baulize: grolle de Landrir ; dewx rases ornés. l'un de méandres en " $\mathrm{H}$ " couchés (urne biconique a col rylindrique). l'aulre de meandres en "s" (jalle a épaulemenl). Ce: dernier motif. que l'on ne rencontre pas en suisse. entre probablement dans la rategorir. des méandres asymél ricpuesz2.

9) Sanjac: grolte des Corbeaux ; un franment a bord carré orné de méandres angulaires et de trails obliques. pale beige orangée follection Caussanel. Villefranche-de-Rouerwue : fig. $11 . n^{\circ} 6$

10-1:, 1,o\%i:Rl: :

10) Balsieges: Lumulus IV du Freysineles: Vase acressoire d'une sépullure a incineralion : panse ovalaire, bord en entonnoir, fond plat: orné sur l'épaule de méandres arrondis ; associe a un vase biconique a col cylindrique ; pas de contexte mélallique (fị. 12. 10).

11) La Malène: Lumulus de Mazel-Bouisssy ; un fragment de pate beige presentant un méandre angulaire excise (collection Prunieres, Mlusee de l'Homme, larisi.

12) Sainle-Enimie: "Lumulus sur dolmen" de l'sistrade : un fragment de paile noire. avee meandre angulaire excise (collection Prunieres, llusere de l'Homme, Paris t.

21) A. Sorrot, In habilal de la cirilisalion des Ghamps alrnes: le siere de la ciranede (Millan. Aeeyron). dans Congres de la Fédéralion des Sore. Acad. du Languedere. Rodez 1958. a paratitre.

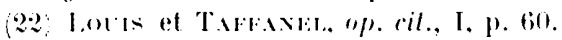

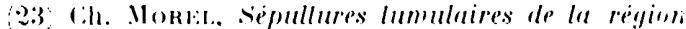

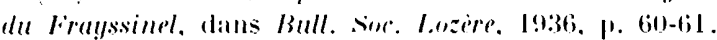




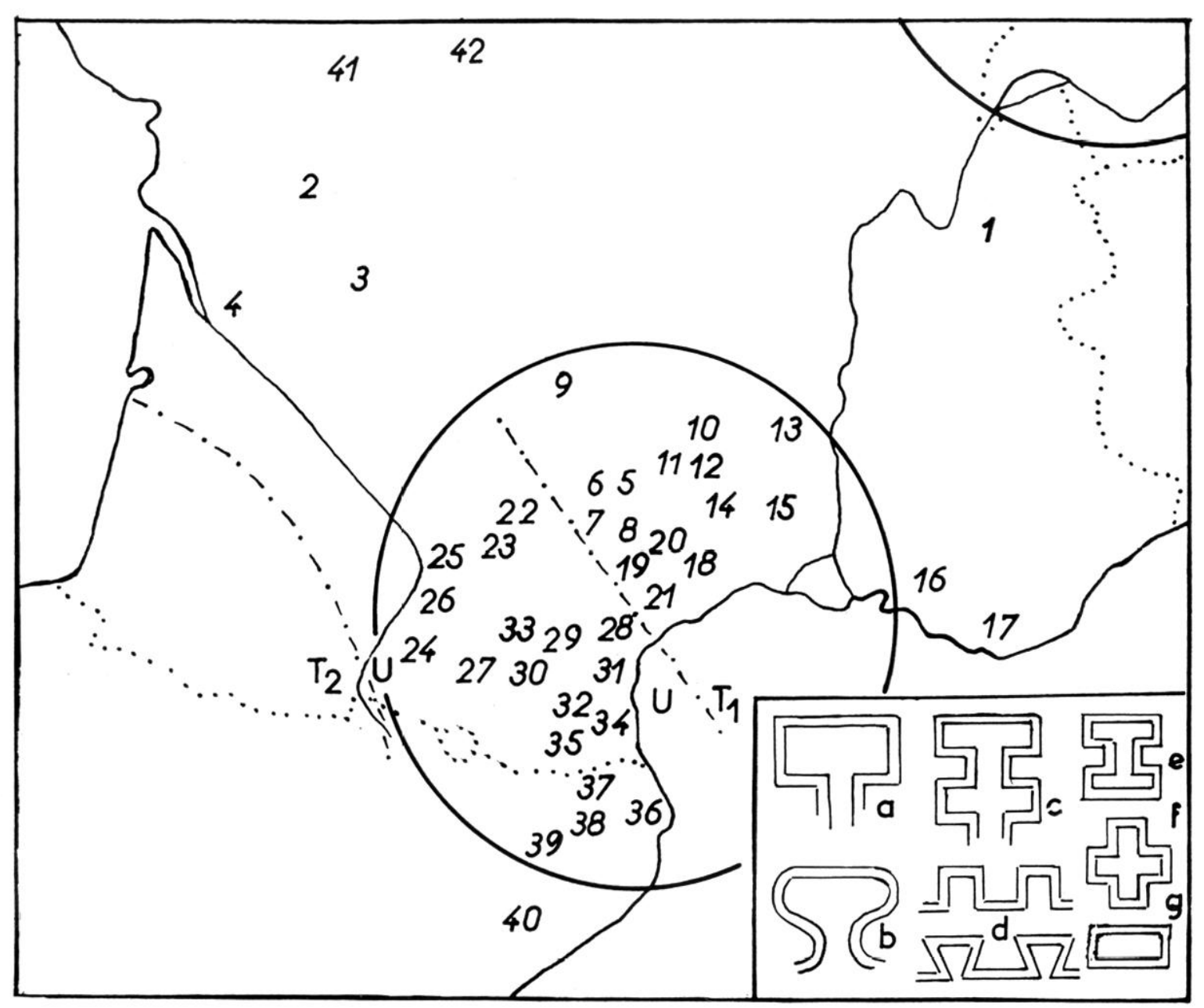

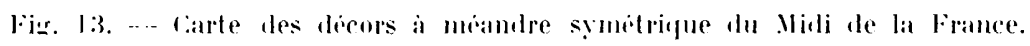

1 : zone des chamus d'urnes: $\mathrm{T}_{1}$. $\mathrm{T}_{2}$ : zone des lumulus.

13 Anuscule Saint-.Marlin-d'Ardeche : grolle des Cloches: plusieurs vases ou fragments ornés de méandres a angles droils a deux méandres rénelés 24 .

\section{4-15) (inRI) :}

14) Lamuejols: erolle de la Fromagerie: fragment d'un vase de mime forme que celui du tumulus IV de Frayssinel (cf. supra, no 10); orné sur l'épaule de méandres angulaires ${ }^{25}$.

15) Nimes: habilal de Languissel ; fragment. orné d'un méandre en " II " couché: d'aulres lessons provenant du mime vase portent des

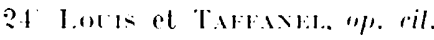

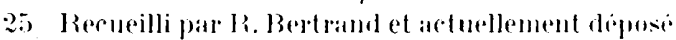
all Nusie de Nimes. décors a struclure asymétrique figuralion stylisée d'animal ${ }^{26}$.

16) Botchts-1) -Rhoss: Istres: oppidam de Saint-Blaise: fragment orné de meandres crénelés i angles aigus 27.

17 Vir. Erenos: habital : 3 Pragment ornés de méandres angulaires. Ie méandre anculaire est associe sur un fragment i un méandre crénele it angles aigus el sur un autre a des impressions triangulaires elles-memes disposées 'n triangle 28 .

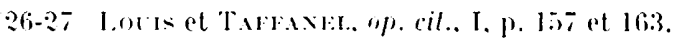

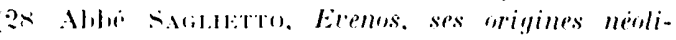
thiques. dans Instilul historique de Pronente. Comgres

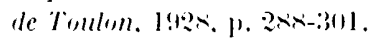


$18-21$ Hinatis:

18) Aniame: habitat du Pont-du-Diable (pour mémoire).

19) Cimssse et leyran: wrolte de MontPeyrous; fragment orné de míandres rectangulaires ${ }^{29}$.

20) Lodere: habilal de Grezac; quelques fragments decorés de méandres angulaires ${ }^{30}$.

21) Saint-Thibery : oppidum de Cessero; quelques fragmenls avec méandres angulaires ont élé trouvés dans la couche la plus profonde ${ }^{31}$.

\section{9-23) Tak :}

2) Puygouzon: Champ d'urnes de LaveneMonsalvi ; fragment de bol orné sous le bord a l'extérieur d'un méandre angulaire ${ }^{32}$.

23) Saint-Sulpice-la-Pointe: Champ d'urnes de (iabor; deux fragment s3 ornés de méandres angulaires tracés soit en lignes incisés réunies par des hachures obliques. soil en lignes ponctués. Le contexte précis de ces deux fragments n'est pas indiqué dans la publication.

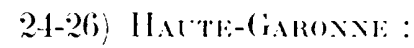

2.1) Gionties: grolle de la sipugo; pelit vase a panse globuleuse orné de méandres angulaires (eollection .J. Molle).

25) Tomlouse: habital du Cluzel; deux menus framments pouvant ilre rallaches aux variétés du méandre angulaire of du méandre crénele (figr. 12,9) ont été lrouves dans les ruines d'une habilation de la premiere période hallstattienne (urne à col cylindrique el panse arrondie, assieltes-couvercles a mulliples faceltes intérieures) ${ }^{34}$.

26) Ténerque: Champ d'urnes de la Trinité; un vase eomplet orné de méandres en " H " couches vient d'itre exhumé au cours d'un labour ${ }^{35}$. C'est une jalte bieonique a

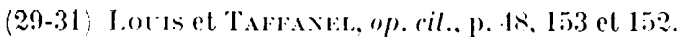
(32) E. Cintanmac, Nole sur l'archeologie prehislorique du deparlement du Tarn, dans .Malériaur..., 1879 , p. 188, fig. 178.

(33) R. Poxtral: ot li. Cinbré, Cimeliere gaulois decouvert ì St-Sillpice (Tarn), dans Revue du Tarn, 1894, p. Iv, no 57 et 59. Rappelons a ce propos, que, contrairement à l'opinion de Déchelette (. Manuel, III. p. 519), la necropole de Gabor est un veritable chanp d'urnes fait de sépultures à incinération en lombes plates, sans aucune trace de tumulus.

(31-35) Fonilles inidites. bord carré, comparable au vase 2 de la lombe 172 de Millas (Pyr.-Or.), dont il sera question plus loin.

27) Ariècie: : Sabaril. enceinle du Peyré; collection J. Vézian, Toulouse.

\section{8-33) AnI): :}

28) Fleury: Champ d'urnes; fragment orné d'un méandre angulaire ; les deux lignes constiluant le molif décoratif sont réunies par des hachures obliques ${ }^{36}$.

29) Mailhac: Champ d'urnes du Moulin; tombe 13, méandre angulaire sur bol tronconique ; tombe 103, méandre double a anoles droits comparable a un motif du Pont du Diable (fig. 11, 3) sur un bol biconique ${ }^{37}$.

30) Mon. : grotte des chambres d'Maric: un fragment de jalle carénée, a bord dégage, ornée sur l'épaule d'un méandre cruciforme (fig. 12,4) associé à un fragment de bol biconique orné d'incisions paralleles (fig. 12. 5). Dans la mime grolle ont élé trouvées so épingles a lide emroulée el une épingle a tibe recourbere. at ajouler à la liste que nous avons dejai dressée. par ailleurs ${ }^{38}$.

31) Varbonne: grotle de Montredon; bol orné exterieurement sous le bord de méandres rectangulaires placés entre deux bandes horizonlales de zigzags incises ${ }^{39}$.

32) Padern: qrolle; fragment de vase a bord presque equarri orné de méandres angulaires $(\text { fig. } 12,1)^{40}$.

33) Pépiente: Champ d'urnes de Las fados. Tombe 32 : vase biconique à col cylindrique orné sur l'épaule de méandres angulaires et associé a une épingle à lèle enroulée. Tombe 36 : vase biconique, a col relindrique, orné sur le col de méandres angulaires el assietlecourerele présentanl des méandres angulaires et rectangulaires ainsi que des motils asymétricues ("marches d'escalier "). Ces deux réramiques ebaient associées a deux pinces a

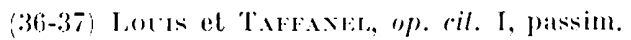

(3); Ce mobilier a ete recueilli par J. Guilaine, rui a bien voulu nous permettre de le mentionner en complement de notre liste (.1. Sorotot, les épingles $a$ lìle enronlee du Midi de la France, dans BSPF, l95!), p. $3315-3.47$.

(39) I.olts et TAFFaxis, op. cil. I, p. 19.

(10) Réserves du Musée de Carcassonne. 
epiler. un raside double à pédoncule el une epingle a tibe platedi.

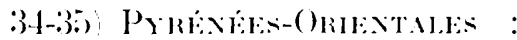

3-1) Combere-les-Cabmules: Champ d'urnes de Reixach : urne ornee de méandres angulaires ${ }^{42}$.

35) Millas: Champs d'urnes. Tombe 26 : méandre angulaire. accompagné d'un zigzag horizontal et de traits obliques. incise sur un vase biconique; parmi le mobilier métallique associe, il y a un rasoir double à pédoncule. Tombe 70: urne biconique a col rylindrique a pied surélevéc3 orné sur l'épaule de méandres doubles. Tombe 1.5; jalle biconique ornée de méandres angulaires présenlant les signes de déginérescence. en ce sens que le méandre perd son caractere équilibre par un allongement d'une des branches ef enferme des lignes brisées (chevrons ef losanges). Tombe 172 : méandre en "Il " rouches incises sur une jatte biconique. Tombe 17:) : méandre angulaire. Tombe 203 : méandre double. 'Tombe $206 ;$ : mème motif.

36-38) Provinee de Gínoxa (Espagne) :

30) Ampurias: Champ d'urnes Parrali; dans la lombe 1, un bol biconique a bord carré, orné à l'exlérieur de méandres angulaires44.

37) I gullana : Champ d'urnes ; dans la lombe 191. appartenant a la partie la plus ancienne de la nereropole. un bol biconique. a pied annulaire. orné à l'exlérieur d'un méandre rénelé ${ }^{45}$.

38) Puerto de la Selva: orotte de los Encalados; fragment orné de méandres angulaires encadres en haul et en bas d'une ligne ponctuée 46.

39-40) Province de Barceloxis (lispagne) :

39) Perafila: crotle de la Eures ; fragment d'une urne biconique a col convexe. ornée de méandres angulaires et de chevrons eqalement incises ${ }^{47}$.

40) Tarrasa: Champ d'urnes de Can Missert, plusieurs vases ornes de méandres a angles droils (lombe li) al de molifs dérivés ${ }^{43}$.

La répartilion géographique du méandre symelrique fail apparailte une zone centrale de crande densile. constiluée par la Catalogne et 171

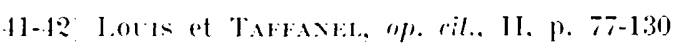

13-1s, lot ts et Taraxis, "p. cii., passim. el le Languedoc. I'Aquilaine. par contre. présente un vide lotal. Landis que la Provence se réduil à quelques points erroupés le long de la còle jusqu'au Var. Il est interessant de noter, de plus. que la zone rentrale coinncide exactement avec l'aire de répartition de l'épingle a lète enroulée que circonscril le rercle reporté sur la carle.

du point de vue archéologique. les points a méandres symétriques se répartissent comme suil :

1) IVabitals de plein air : 11 poinls ( $n^{\text {os }} 1.2$. 6. 14, 16, 17, 18,20, 21, 25, 27);

2) Cirotles : 15) points (nos 3, 4. 5. 7. 8. 9, 13. 15. 19. 24. 30, 31. 32, 38, 391.

3) Sépultures : 1.4 points (no 10, 11, 12, $22,23,26,28,29,33,34,35,36,37,40)$.

S'il est dilficile dans la plupart des ras, faute d'observations sullisantes, d'inlerpreter les trouvailles faites, dans les habilals el surtoul. dans les grolles (nécropoles. habilats permanents, refuges temporaires, lieux de culte?), par contre, l'examen des sépullures permet de conslater que, dans dix cas sur lreize. le méandre symétrique est lié à des incinérations en lombes plates el. dans trois a as seulemenl, à des lumulus, dont un $\left(\mathrm{n}^{\circ} 10\right)$ à incinération. Il est donc légitime de penser que ce déor caracléristique a elé introduil dans le Vidi de la France par la civilisation des Champs d'urnes el qu'il s'est irradié dans la zone des lumulus, oi il est principalement représenté dans les groltes et les habilats. Comme dans le cas de l'épingle à tele enroulée. les points geographiques se répart issent sur la zone des Champs d'urnes proprement dils. qui. en l'élat actuel de nos connaissances, ne dépasse pas vers l'Est le cours de l'llérault. el sur la zone d'influence de retle civilisation, all Nord-Est de ce departement. Ia présence en Lozere nos $^{\text {os }} 11$ et 12) du méandre symétrique sur de la céramique excisee hallstallienne, particulierement typique de la civilisation des lumulus du sudEst du Massif Central+99. souligne les conlacts

49: Carte de ripartition dans A. Soltor, I.a

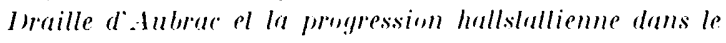
sind du .Massif Central dans lahiers l.igures darchendugie el de prehishive, 1959, no 8. 1. 41, liy. 2. 
qui se sont opérés dans le Midi de la France entre les deux rivilisations fondamentales du premier lege du ber.

sur le plan chronologique. enfin, si le méandre scrmélrique apparail en suisse dise le débul du Bronze linal III of disparail is

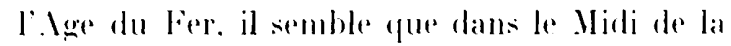
France son applarition el sal disparition soient un peu plus lardives so. Comme nous avoms pu

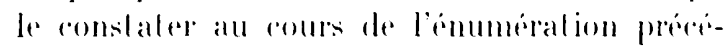
dente. il est assorie dans plusieurs miliend an el parfois sur un mime vase. areer des molits différents tiguralions slylisées de persomnages humains of d'animaux dont X. K. Sandars a clabli l'origine of la datation approximalive. Ces motifs. comme d'ailleurs la ereecque asymélrique. proviennent de la Médilerranéte orientale fdou ils sont parrenus sur le sol francais par l'inlermédiaire de l'llalie du sud: el ne sont pas antérieurs is la fin du vine sierle avant J.-C. La carte de répartilion de ces figurations slytisésose montre bien que rese molifs se rencontrent principalement sur la cole méditeraneenne. a lexception de lrois point: silues a l'interieur de la France : vase d'une palafitle de Chatillon. sphéreside de bronze du dépol de la Ferté-llauterive. assietlecouverete d'un lumulus de Villement. Dans: a dernier cas. la céramique. qui est approximalivement datée par l'épée hallstaltienne de bronze trouvere dans un lumulus voisin. présente a la fois. le méandre symétrique el les figuralions slylisées. Il est done vraisemblable de penser que. dans le Midi de la France. le méandre symétrique en provenance du Cord-bis s'est prolongé juspu'a l'expansion plus lardive des figuralions styliseses. Commente res deux ablegories de molifes sont traitíns suivanl la meme lorhnique (incision an simple. double ou triple trail il esl probable gue cess deux groupes. qui alleignent sepalrement al par des voies diflérentes le Midi de la Franere. sont fondamentalement apparentes el proviennent peruteilde d'un berefau commun

(5) Il faut tenir compte llu fail que de Bromze Final de la suisse est. en pattie. synehronique du Hallstatt $I$ de la fiance, comme l:a expressiment souligni Dechelette (Mamuel. III. 1. 3titi.

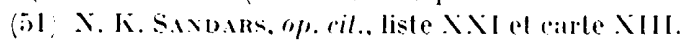

silue dans le sud-bel de l'Europe : leurs hemins dexpansion convergeraient dans le Centre el le vlidi de la France oid. dies lors. les deux déeors seraient par endroils contemperains: aros nolammenl le ras al Millats oil les mimes rases (jalles bienigues portent soil des méandres simelrigues (lombers 2019 al 172. soil des ereecpues ou des liguralions sylisées (lombe losi. La lombe 70 du mimn cimetiere conlient mime une urne particu-

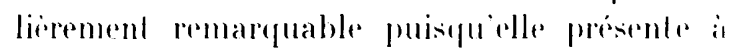
la fois un décor à méandre symét rigure originaire du Vord-list et une lorme imilér d'un vase de bronze originaire du sud-list 52.

1 en juger par le sile le mieus iludie Caillar. lude. le décor à méandres symetriques. que l'on reneontre dans l'habilal du Cayla I el dans la nécropole correspondante du Moulin. disparail au débul de la seconde:

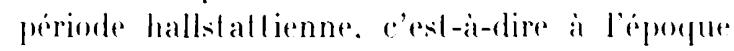
des poignards a antennes: on me le retrouve plus sur la réramique des lombes du cirand Bassin I (a) Mailhar: non plus que sur relle du Cayla II el du Cirand Bassin II. Bien rntendu. ces observations ne sont valables que pour le Vlidi de la France: dans d'aulres régions. nolamment dans l'bil. Ie méandre: simétrique dure tout an long du premier I I du Fer juscula la l'ine ${ }^{33}$. Dans l'Europe rentrale. en particulier en Boheme ol en silesie. il fail miome partie du répertoire décoralif

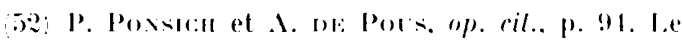
vase yui a puservit de modele provient de lappuinies

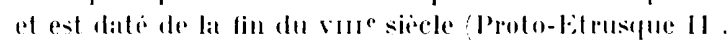
liurne de Millas lombe za est considerée comme le plas ancient vase de la medepole. Comme mons lat fait

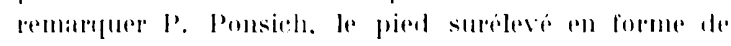

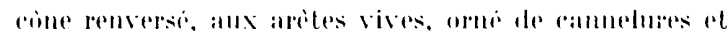

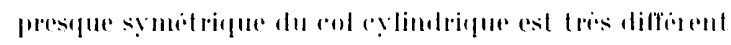

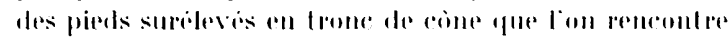

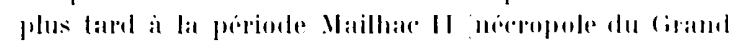

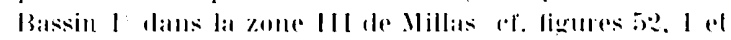

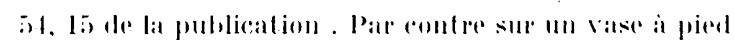

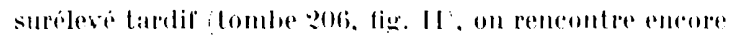
int mamble symetriplue.

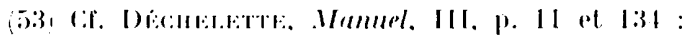
vase du tumulus de Diarville a panse arrombe el horel vertical en "fatc-col "Kratentand : vase du tumulus du Bois de langres associe à une epee de fer hallstatlienne et a un rasoir semi-cireulaire a lumieres. gravi all frémolo. Vases de la Tene de la collection Vorel an British Wuseum finide lo Varly Iron Alge Anliquilies. l.ondres. 19.25, pl. 5. 1 . 


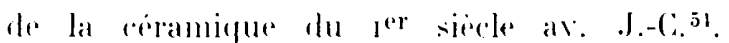

I. associalion sur un fragment du Pont du Diable de meandre svmelrique incise el des: rannelures légeres est contirmée par la presence simulanée. dans un meme niveau de la sorolle. de la Roque-saint-Christophe f point no $3:$ : de deux vases a cammelures légeres of d'un lesson a méandre incisé. Ce niveau. dalé par ․ K. Sandars de la fin du Bronze Final ll ${ }^{53}$. contenail éalement un fragment de poterie rhamplevere (Kerlschnill) du lype de l'Age du Bronze. bien differente de la réramigue. champlevée des lumulus de l'Ige du Fer donl nous arons deja parle. qui appartient a une calégorie de céramigue anterieure aux Champs d'urnes. La juxlaposilion sl raligraphiepue de res trois esperes de poterie indique sans: doute que le meandre symétrique el la ramnelure légere raraclérisent la réramigue de la premiere vague de la rivilisalion des Champs d'urnes. qui. dans le Midi de la Fancese se substilue a l'ancienne civilisalion de l'age du Bronze donl la reamigue rhamplevée. yui remonle all Bronze lloyen. se prolonge jusqu a la veille de l'iege du Fer (Mallstall B de Reinerlie:

Le méandre srmetrique permed dome de dater aver assez de précision ${ }^{50}$ Jarrive de la premiere vague de la rivilisation nouvelle qui déferle. d'un seul eoup. juscpue dans la région de Bareelone Tarrasa. Cel emahissement est jalomne par les grandes néropoles des plaines languedordennes Mailhar. Pépieus el des vallees ralalanes (Millas. Inullana. Mors que dans les régions monlagneuses de la bordure sud du Massif Central on ne peul

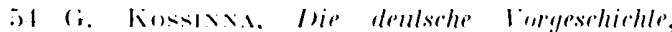

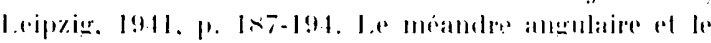
meandre remele sont fres alomblamment pepresentes

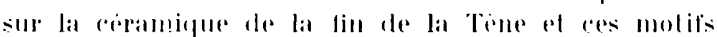

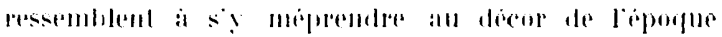

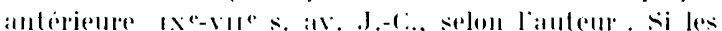

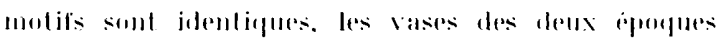
prosentent natmerllement des formes lout a fait diffilontes.

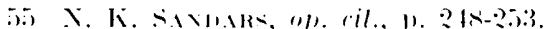

sti lovase de la fombe

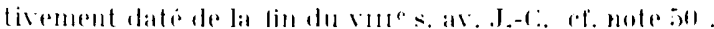

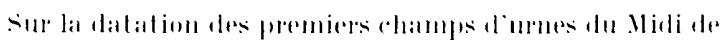

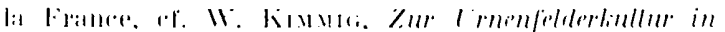

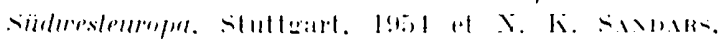
ips. ril. e rh. VIII. romstater que des inlluencess exlépienere modiliant plus ou moins les roulumes des populations anterienres qui restent loujours majorilaires. il semble que dans le comber languredecien. entre le llassif Central el les Prenés. il ne sagisie pas de simples infill ralions mais de: lomplantation de eroupes elhniques oreanises. yui praliquent leurs collumes funéraires I radilionnelles (Champe durnes vérilabless al fabriquent leurs poleriess caracléristiques, loul a lail diflérentes de la réramique emplovere jusefue-lic dans le pays qu'ils ont oceupé.

Dans le cercle malerialisant la zone de plus srande densilé du meandre symét rique. saujac. dreyron fige 13. no 9. constitue le poinl extreme vers le Vord-(luest. Ce gisement fait pour ainsi dire la liaison entre le eroupe languedocien-ratalan el les trois points de l'ouest du Massil Central nos 2. 3. ol t: anquel. il faudrail ajouter pour mémoire. bien qu'il ne soil pas silue dans la région que nous etudions. lhabilal de sous-Clan JaunayClan. Vienne. $n^{0}$ tl 58 el le lumulus de Villement chaint-doustrille. Indre. no $4: 2$ dont nous avons parlé. I sous-Clan. nous rel rouvons. en elfel. quelques varietes lypiques de méandres scmelriques incisés: méandres angulaires. meandres rectangulaires (variante du lype $g$ el méandres crénelés a angles oblus emboiles les uns dans les autres Varianle du lype d:5x. Ces fragments décores signifienl que le débul de cel habital remonte. comme au Pont du I liable. a une periode qui peut s'elaler sur le Bronze final III of le Ilallstall I de béhelelle: Enfin. la position géographique de: sous-Clan semble indiquer que la dilfusion du méandre symélrique vers le Vlidi de la France sest elleceluée principalement par louest du llasid cientral.

linsi les lessons du Pont du lliable permeltent de fixer un ensemble remanique pouvant itre all ribue a une période encore mal comnue de la probohistoire du Midi de la France. Celle reramique. cont nous arons note quelques déments lypologeigues bien definis décor

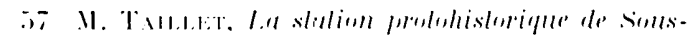

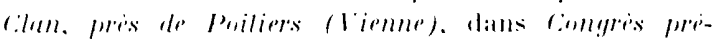

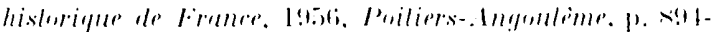
113.

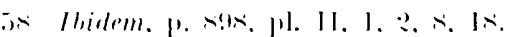


incisé à méandre symél rique, cannelures léqueses, bords carrés avec ou sans rainures sur la tranche supérieure 59. est contemporaine de la vague des Champs d'urnes qui. a la fin du Bronze final. alleinl pour la premiere fois. d'une maniere massive. le sud-ouest de la France el la Calalogne. aprés aroir pénétró au cours des périodes prérédentes dans l'list el le Centre-Sord de la france. Comme la plupart des pieres manquees appartiennent a ce eroupe réramique du Bronze final III, il semble assuré que, dis relle époque. un erompe appartenant a la civilisalion des Champs d'urnes. ou loul au moins fortement inlluence par celle civilisalion. élait inslallé a Iniane prís de Saint-.Jean-de-Fos, el y fabriquail deja sur place sa polerie.

Finalement l'histoire de l'habitat du Pont du Diable semble se présenter ainsi :

1. Installation a la fin de l'Age du Bronze d'un eroupe humain porleur d'une poterie elrangere a la région foivilisation des Champs d'urnes).

II. Ce groupe se mainlient sur place loul au long du Premier Ige du Fer.

III. lans les periodes suivantes. par suile peut-ìtre d'un déplacement de l'habilat principal, le site n'est plus que fablement occupe. A l'époque romaine il semble avoir eté déserte, ef l'on peut supposer que les dernieres rases qui subsistaient sur le haul de la pente ont servi de lieu de cachelle ou de refuge dans les temps lroubles du Bas-Empire al des grandes invasions.

IV. Au Moven Age. les pentes du Pont du Diable sont definilivement abandomners of les poliers s'installent, a l'époyue historique, a quelques renlaines de melres plus a l'ouest des parois rocheuses pour balir le village de saint-Jean-rle-Fos.

Malgré loules les vieissiludes de sa longue histoire, le teroir avoisinant possédail loujours la mème argile qui avail élé deja utilisée au débul du ler millénaire av. J.-C. : r'est celle mème matiere premiere qui. laconné une vinglaine de siecles plus lard par de's poliers languedociens. fera connaîl re ce petil village dont nous arons tenté, à la lumiere de quelques lessons modestes mais précieux, de jalomner le developpement au cours des àges.

Jules Boctoot, Jean Aris., André soctou.

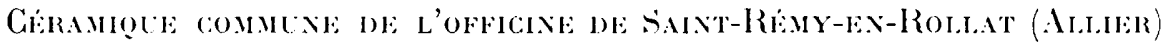

L'oflicine de poliers gallo-romains de SaintRemy-en-Rollat est bien connue depuis le

(59) I.es motifs incises semblent le plus souvent associcis aux formes suivantes :

a) bol ou jalte hiconique a carine plus on moins marqué \{figr. 11,3\}; Mailhac. Millas. Pont du Diable; b) vase a panse ovalaire et hord en entonnoir (fi 2.2 .10 : balsioges, lammojols. Cette mime forme se retrome a St-Ien-sur-ligere (А. К. SANmars, op. cil., p. 25, fiur. 69, 5, ;

c) bol tronconique (ligr. 12, 9) : Singen, Mathate; d) vase hiconique a col eylindrique : Pépieux.

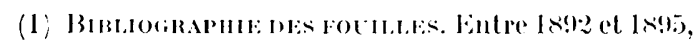
un proprictaire de saint-Rémy-en-lhollat, II. Civois, trouva dans ses champs des fosses remplies de dibris céramiques, constituant raisemblablement des depotoirs d'ofricine de potiers qallo-romains. Il les vida sans beaucoup de soin, conservant les pieres qui lui semblaient remarquables. Au cours des recherehes yuil fit ainsi, il amassa une assez belle collection fournal persomnel de Bertrand, archéologrue moulinois, aima- travail que lui a consacré Decheleller. Flle est

blement depouille par .1. Gimud-lavergne. qui le détient. En 1900, lors d'une excursion de la société d'limulation de l'Allier, becheletle et Bertrand achetierent la collection de M. (iivois (A. Brotraxis, Bulletin de la socirti alimulation de lallier. 1900, Chrmique, p. $207-30 s ;$ et notamment un dibris de vase a relief dipplique, recouvert de whacure jaumatre, representant le combat de Thesio of d'tlippolyte

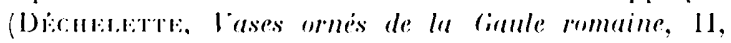
p. 19:-19s, $n^{\circ} 22$, . un rhyton a deux tetes, vernis vert signe dITTls, ibid. I, p. 12, , un grand vase forme 62 (ibill., I, p. As, tig. 11 et pl. I), des statuettes en terre blanche tris diverses, restees jusqualor's incilites (Journal de Bertraml, des fragments de vases rouges rernis et de vases noirs. lin 1900, Dechelette et Bertrand font une campagne de fouilles fructueuses en vase et en figurines, sur un termin voisin encore vierge (A. Bertraxis, chronique citie). Dechelette empechi, Bertrand surveille seul les recherches, et rédige un compte rendu, sans plan, qui parait sous une double 